\title{
Preparing the Next Generation of Academic Researchers During the Pandemic: Lessons from a National Mental Health Research Postdoctoral Fellowship
}

\author{
Nathan C. Hantke ${ }^{1}$ (D) V Viktoriya Samarina ${ }^{2}$. Joachim Hallmayer ${ }^{3}$ - Lauren Anker ${ }^{3} \cdot$ Ruth O'Hara $^{3}$. \\ Sherry A. Beaudreau ${ }^{2}$
}

Received: 22 September 2021 / Accepted: 18 February 2022 / Published online: 7 March 2022

(C) This is a U.S. government work and not under copyright protection in the U.S.; foreign copyright protection may apply 2022

\begin{abstract}
Objective The COVID-19 pandemic has severely disrupted all aspects of academic medicine, including post-doctoral research fellowship training. The current survey examined ways in which research fellows across 28 U.S. nationally diverse sites have been impacted.

Methods Survey participants included 62 M.D. and Ph.D. post-doctoral fellows and 27 local fellowship center directors within the Veterans Affairs (VA) Advanced Fellowship in Mental Illness Research and Treatment (MIRT), a national fellowship program tasked to develop academic clinician researchers within the field of mental health. Survey questions focused on productivity and challenges experienced by fellows during the pandemic.

Results Half of fellows reported working entirely off-site during the COVID-19 pandemic. All fellows reported some level of disruption in productivity during the pandemic; 73\% reported a disruption in data collection, $69 \%$ reported decreased scholarly output, $41 \%$ reported disruption in grant writing, and $73 \%$ reported disruption in ability to provide clinical care. Yet, the majority of fellows (66\%) reported not having to change their research goals, pivoting to telehealth-based data collection, and employing extant data for research projects and peer-reviewed publications.

Conclusions The results of the fellow and director surveys highlight the associated disruption of the COVID-19 pandemic on fellowship-related activities and parallel ingenuity of programs to continue conducting research and clinical services in a modified fashion. While many research goals continued unabated, the findings suggest alterations in data collection methodology and a focus on using extant data, which may have a residual influence on future early career research grant applications.
\end{abstract}

Keywords COVID-19, Post-doctoral fellowship · Research education

The abrupt onset and prolonged state of the COVID-19 (SARS-COV-2) pandemic has radically altered medicine. Clinical care, research, and training have experienced significant disruption, with a call to balance safety and well-being [1]. With regard to training future researchers and clinicians, residencies and fellowship programs have pivoted from standard in-person curricula to virtual formats to decrease possible virus exposure and spread [2]. These alterations have had far-

\footnotetext{
Nathan C. Hantke

Hantke@ohsu.edu

Oregon Health \& Science University, Portland, OR, USA

2 Veterans Affairs Palo Alto Health Care System, Palo Alto, CA, USA

3 Stanford University School of Medicine, Stanford, CA, USA
}

reaching implications for trainees. Providing clinical rotations, recruiting trainees, and maintaining accreditation standards all pose problems for training sites during the pandemic [3].

In addition to programmatic disruption, the pandemic has adjusted fellows' subjective training experience. A recent large survey in Nature [4] indicated general feelings of concern for a stunted career trajectory among post-doctoral fellows. Eight out of ten postdoctoral fellows reported COVID19 has had a negative impact on their ability to conduct experiments or collect data.

Understanding continues to evolve regarding the immediate and potential long-term impact of the COVID-19 pandemic on fellowship programs and trainees. The ways in which large multisite programs with shared training goals have adapted during the pandemic are not well documented, yet have potential to provide significant insight into the disruption 
and related adaptation of research fellows on a national level. The current needs assessment surveyed directors and fellows within the Veterans Affairs (VA) Advanced Fellowship Program in Mental Illness Research and Treatment (MIRT), consisting of 28 unique fellowship sites at 24 locations across the USA. The survey examined ways in which mental health research sites have pivoted over the past year and focused on ways programs have continued to be productive throughout the pandemic.

\section{Methods}

Survey participants included post-doctoral fellows and local fellowship center directors within the VA MIRT, a 2-year fellowship program tasked to ensure the future of the academic pipeline of clinical researchers within the field of mental health [5]; fellows may apply for a competitive third year of funding. This fellowship program encompasses the Mental Illness Research Education Clinical Centers (MIRECC), Centers of Excellence (CoE), Serious Mental Illness Treatment, Resource, and Evaluation Center (SMITREC), Centers for Integrated Healthcare (CIH), Centers of Excellence for Stress and Mental Health (CESAMH), and National Center for Posttraumatic Stress Disorders (NCPTSD) programs. Fellows spend $25 \%$ of their time providing direct clinical services to Veterans, with the remaining $75 \%$ allocated for clinical research and grant writing-related activities, and didactics. Each fellowship site recruits one M.D. and one clinical psychology Ph.D./ Psy.D. trainee annually, and has one to two M.D. directors who oversee the local M.D. fellows and one to two Ph.D. directors who oversee the Ph.D. fellows. Fellows have dual appointments at VA and the associated academic institution [5]. A national coordinating center for the program has ensured the continued success of fellowship sites through administrative support, curriculum development, and other professional development resources offered to fellows (e.g., consultation on genetics research, neuroscience, clinical trials, grant writing, and curriculum vitae).

Fellows and directors completed unique surveys (available on request from the corresponding author) aimed at determining the impact of the COVID-19 pandemic on fellow progress within the fellowship program, with a mixed method design of quantitative and qualitative questions. Specifically, directors and fellows were asked to estimate the extent to which fellow productivity decreased on different metrics. For example, respondents were asked to provide their percentage of time currently spent onsite versus working remote. Other questions required respondents to determine perceived disruption in productivity in defined areas using Likert-type scales or multiple choice. Survey administration and collection was via an online cloud-based survey software (Qualtrics and Survey Monkey) and disseminated via email directly to local center directors and current fellows within MIRT. The director survey was open for responses from August 24th to September 4th, 2020, about 5 1/2 months after the March 13th, 2020 U.S. COVID-19 national emergency declaration. The fellow survey was open for responses from January 22nd to February 10th, 2021. To ensure anonymity, directors and fellows did not provide demographic information in survey responses. The VA Portland Health Care System Institutional Review Board reviewed this survey and determined it to be consistent with Quality Assurance/Quality Improvement, with no required IRB approval.

\section{Results}

Twenty-seven fellowship directors completed the survey, reflecting a 55\% response rate. Sixty-two fellows completed the survey, reflecting a $77 \%$ response rate and included 26 first-year, 25 second-year, and 11 third-year fellows.

Only one director reported a delay in fellow start date due to the COVID-19 pandemic. Fellow time spent working remotely varied from 0 to $100 \%$ depending on fellowship location, with an average of $89 \%$ of fellow time spent working remotely across all sites. Specifically, $78 \%(n=21)$ of fellowship sites reported fellows spending between 80 and $100 \%$ of their time working off-site during the pandemic. As of the fall of 2020, $96 \%$ of directors reported planning to sustain this work set-up throughout the duration of the pandemic, a statement supported by the February 2021 fellow survey described below.

Across all sites, directors reported subjective estimated decreases of an average of $41 \%$ in fellow productivity in data collection, $6 \%$ in scholarly output, $2 \%$ in grant writing, and $15 \%$ in providing clinical care during the pandemic. All directors reported that fellows at their sites continued to make progress towards fellowship goals, primarily accomplished by converting data collection to virtual modalities, working with archival data, and focusing on manuscript writing. Directors qualitatively reported concern about stalled fellow pilot studies, including small grant-funded projects, and the long-term impact on fellows' competitiveness for early career research grants.

Among fellows, $50 \%(n=36)$ reported spending $100 \%$ of their time off-campus during the pandemic, with a range of $10-100 \%$ depending on fellowship location, equating to an average of $90 \%$ time working remotely across all fellow respondents.

Among respondents, 34\% ( $n=21)$ of fellows reported having to change their research goals due to the pandemic. Most fellows $(56 \% ; n=35)$ reported interrupted or slowed progress towards fellowship goals during the first 10 months of the pandemic, but $37 \%(n=23)$ reported that their goals remain uninterrupted. Six percent $(n=4)$ of fellows reported severely interrupted progress on fellowship; 3 of those 4 respondents 
were first-year fellows about 6 months into their fellowship. Forty percent of fellows $(n=25)$ expressed concern that the pandemic has negatively affected their career prospects, while $32 \%(n=20)$ were unsure of the impact and $27 \%(n=17)$ believed there will be no negative impact; responses were distributed across first-, second-, and third-year cohorts.

Regarding daily fellowship-related tasks, $73 \%(n=45)$ of fellows reported disruption in data collection, particularly so for second-year $(76 \%)$ and third-year $(81 \%)$ fellows. Sixty-nine percent $(n=43)$ of fellows reported disruption in scholarly output, $41 \%(n=26)$ reported disruption in grant writing, and $73 \%$ $(n=45)$ reported disruption in providing clinical services. Specifically, fellows on average perceived a $36 \%$ decrease in productivity for data collection. The average perceived decrease in productivity was $18 \%$ for scholarly output, $12 \%$ for grant writing, and $19 \%$ for ability to provide clinical care. For adjustments made by fellowship programs, $76 \%(n=47)$ of fellows reported switching to telehealth for ongoing studies, 53\% $(n=33)$ reported switching to secondary data sets while primary data collection was halted, and $10 \%(n=6)$ reported making no adjustments in response to pandemic-related delays in conducting research. Directly comparing fellow and director subjective report, directors reported slightly greater disruption in clinical care (10\% greater) and data collection (5\% greater), while fellows reported slightly greater disruption in scholarly output (12\% greater) and grant writing (10\% greater).

Eighty-one percent $(n=22)$ of first-year fellows reported receiving clear guidance of how their program will provide them support during the pandemic. Thirty-two percent $(n=20)$ of all fellows reported that sites created formal social events, either virtual or with limited in-person contact to foster a sense of community during the pandemic. Fifty-two percent $(n=32)$ of fellows reported attending site-facilitated, group-based, professional development meetings (e.g., journal clubs) designed to foster camaraderie. Thirty-four percent $(n=21)$ of fellows reported engaging in activities other than those listed above to create a sense of community, such as biweekly fellows-only meetings.

Survey responses yielded largely consistent peer-to-peer advice (e.g., fellow advice for other fellows) for navigating pandemic-related disruption. Director-to-director advice themes emphasized the importance of communication with the IRB, providing logistical support for fellows working remotely (e.g., VA issued laptops), and frequent check-ins with fellows. Fellow-to-fellow advice themes emphasized being flexible, creating contingency plans for data collection, and seeking ways to socialize online with peers, such as a joining a writing group. Fellow-to-director feedback themes described their appreciation of their director's advocacy for fellow safety during the pandemic, their encouragement of professional networking to prevent isolation and to receive guidance from more senior fellows, their acknowledgment of the altered dynamic of providing mentorship over video, and their director's mindfulness of symptoms of burnout.

\section{Discussion}

The COVID-19 pandemic had significantly disrupted day-today operations for all surveyed fellowship programs. Yet, based on survey responses, directors and fellows reported successfully adapting with continued progress towards fellowship goals primarily by using telehealth services.

Pandemic-related disruption varied across fellowshiprelated tasks. Both directors and fellows reported greatest disruption in data collection and clinical care. A large portion of fellows reported disruption in data collection, particularly for fellows in the second- and third-year cohorts, in which pilot data collection is vital for early career grant submissions. However, most fellows were able to continue research projects by using alternate data sources (e.g., extant data sets) or pivoting to telehealth-based data collection. Similarly, $73 \%$ of fellows reported subjective disruption in providing clinical services, but only a $19 \%$ decrease in clinical productivity. This continued productivity was accomplished by embracing telehealth services, suggesting this modality holds promise for mitigating pandemic-related fellowship disruption in the future. Findings suggested grant and manuscript writing lends well to working remotely; perceived productivity in these areas was negatively impacted by the pandemic, but much less so than data collection or clinical services.

Except for fellows reporting greater perceived decreased productivity in scholarly output and grants, fellows and directors reported comparable subjective declines in fellowshiprelated productivity despite a separation of 5 months between surveys. There are many possible reasons for this finding, such as stabilization in the ability for data collection and clinical services via telehealth following initial early disruptions, a perceived burden of disruption in scholarly output by fellows as the fellowship progresses, or potential differences in priorities of stakeholders. This continuity of findings is important, as directors were surveyed about 5 months post-national emergency declaration, but prior to the vaccine rollout in the USA, while fellows were surveyed an additional 5 months later during the initial vaccine rollout for older adults and healthcare workers. It is not surprising that findings did not significantly vary, given the vast majority of medical centers and research labs continued modified operations during both surveys. These survey findings are consistent with other reports of a shared experience across academic and medical settings, with an initial halting of in-person data collection, substantial research protocol revisions, and pivoting to remote data collection during the initial months of the pandemic, resolving into a modified, yet stable fellowship experience $[6,7]$.

The long-term impact of the pandemic on the trajectory of early career M.D. and Ph.D. mental health researchers remains unclear. Despite significant disruption, respondents reported slowed yet continued progress towards fellowship goals, which is promising. However, many fellows reported pivoting their 
work to telehealth-based data collection or secondary data analysis, which has the potential to bias future proposed grants towards telehealth-based studies, or have other unforeseen consequences. In addition, most fellows expressed concern or feeling unsure about how the COVID-19 pandemic may negatively impact career prospects. Most fellows reported feeling supported by their local fellowship program, but responses suggest the presence of some uncertainty and anxiety about the future.

Several possible limitations of these findings are worth noting. First, survey results are based on the opinions of fellows and fellowship directors during a 6-month span of the pandemic. As a potential focus of future program evaluations, longitudinal monitoring of objective measures of research productivity (e.g., grant obtainment) could provide a more granular analysis of the key differences that drive continued or disrupted productivity and outcomes during the pandemic for early career mental health researchers. Second, respondents were all within the same overarching fellowship program (VA MIRT); findings might differ for postdoctoral programs in other settings, such as state-sponsored or private universities. However, the findings also represent a national cohort across different stages of fellowship within very different VA and academic affiliate settings, which is a particular strength of the study and adds external validity. Third, respondents encompassed mental health research fellows, who spend the majority of their time engaged in clinical research and only spend $25 \%$ of their time providing direct clinical services. The sample provides a crucial perspective into the impact of the COVID-19 pandemic on both research goals and clinical services for M.D. and Ph.D. fellows in the field of mental health, but may not extrapolate to other fellowship programs focused on different disciplines. The surveys did not query about gender, race, or ethnicity, or the impact of personal stressors during COVID-19 (e.g., childcare or other family caregiver roles), which are factors speculated to lead to differential experiences in fellow productivity and disruptions in a research fellowship. Given the documented challenges that women and ethnic and racial minorities faced in academia, even prior to the pandemic, future surveys examining these factors would likely be of importance for delineating the complex impact of COVID-19 in academia [8].

In summary, survey results highlight the associated perceived disruption of the COVID-19 pandemic on fellowship-related activities and parallel ingenuity of programs to continue conducting modified research and clinical services. While many research goals continued unabated, findings suggest alterations in data collection methodology and a focus on secondary data to support fellow research productivity, which may have a residual influence on future early career research grant applications. Survey responses suggest that fellows were most successful when able to quickly modify their studies to allow for mental health research data collection via telehealth, suggesting the possible utility of establishing contingency plans that allow for fellows' studies to convert to telehealth to mitigate future disruption in data collection. Lastly, survey findings have implications for the critical role of director support to weather these challenges by establishing these data collection contingency plans, by creating opportunities for fellow socialization, and by offering wellness check-ins to provide potential support for burn-out.

Funding Writing of this manuscript was supported by the Department of Veterans Affairs Office of Academic Affiliations. Views expressed in this article are those of the authors and are not necessarily those of the Department of Veterans Affairs or the Federal Government.

\section{Declarations}

Disclosures On behalf of all authors, the corresponding author states that there is no conflict of interest.

Ethics approval The survey was reviewed by VA Portland Healthcare System Institutional Review Board and determined to be consistent with Quality Assurance/Quality Improvement, and thus did not require IRB approval.

\section{References}

1. Chau KH, Nouri SN, Madhavan MV. Fellowship in the time of coronavirus disease 2019 (COVID-19): a time to adapt. JAMA Cardiol. 2020 Jul 1;5(7):749-50.

2. Sanches M. Research Education, Distance Learning, and the COVID-19 Era. Acad Psychiatry. 2021;45:639-40.

3. Potts JR 3rd. Residency and fellowship program accreditation: effects of the novel coronavirus (COVID-19) pandemic. J Am Coll Surg. 2020 Jun;230(6):1094-7.

4. Woolston C. Pandemic darkens postdocs' work and career hopes. Nature. 2020 Sep;585(7824):309-12.

5. O'Hara R, Cassidy-Eagle EL, Beaudreau SA, Eyler LT, Gray HL, Giese-Davis J, et al. Increasing the ranks of academic researchers in mental health: a multisite approach to postdoctoral fellowship training. Acad Med. 2010 Jan;85(1):41-7.

6. Ghosh S, David JG, Amaro CM, Gaultney WM, Schmidt AM. Commentary: "How's it going?" training experiences of pediatric postdoctoral fellows during COVID-19. J Pediatr Psychol. 2021 Feb 19;46(2):144-52.

7. Stiles-Shields C, Plevinsky JM, Psihogios AM, Holmbeck GN. Considerations and future directions for conducting clinical research with pediatric populations during the COVID-19 pandemic. J Pediatr Psychol. 2020 Aug 1;45(7):720-4.

8. Galley HF, Colvin LA. Next on the agenda: gender. Br J Anaesth. 2013 Aug;111(2):139-42.

Publisher's Note Springer Nature remains neutral with regard to jurisdictional claims in published maps and institutional affiliations. 